\title{
Soret and Dufour Effects on Transient Double Diffusive Free Convection of Couple-stress Fluid past a Vertical Cylinder
}

\author{
H. P. Rani ${ }^{1 \dagger}$ and G. J. Reddy ${ }^{2}$ \\ ${ }^{1,2}$ Department of Mathematics, National Institute of Technology, Warangal, AP, 506004, India
}

†Corresponding Author Email: hprani@nitw.ac.in

(Received April 19, 2012; accepted November 15, 2012)

\begin{abstract}
This study examines the influence of Soret and Dufour effects on double diffusive transient free convective boundary layer flow of a couple-stress fluid flowing over a semi-infinite vertical cylinder. A set of non-dimensional governing equations namely, the continuity, momentum, energy and concentration equations is derived and these equations are unsteady non-linear and coupled. As there is no analytical or direct numerical method available to solve these equations, they are solved by using the CFD techniques. An unconditionally stable Crank-Nicolson type of implicit finite difference scheme is employed to obtain the discretized forms of governing equations. These equations are solved using the Thomas and pentadiagonal algorithms. The numerical results are compared and found to be in good agreement with previously published results as special cases of the present investigation. Transient velocity, temperature and concentration profiles, average skin-friction, Nusselt number and Sherwood number are shown graphically for different values of Soret (So) and Dufour (Du) numbers. In all these profiles it is observed that, as the values of So decreases or the Du increases, the time required to reach the temporal maximum and the steady-state increases. It is also observed that the average values of skin-friction and heat transfer rate increases with the increasing values of So or decreasing values of Du. Whereas the reverse trend is observed for the average mass transfer rate.
\end{abstract}

Keywords: Soret and Dufour effects, Couple-stress fluid, Natural convection, Vertical cylinder, Finite difference method.

\section{NOMENCLATURE}

$\mathrm{Bu} \quad$ combined buoyancy ratio parameter

$c_{p} \quad$ specific heat at constant pressure

$c_{s} \quad$ concentration susceptibility

$C^{\prime} \quad$ species concentration

$C \quad$ dimensionless species concentration

$\overline{C_{f}} \quad$ dimensionless average skin-friction coefficient

$C_{f} \quad$ dimensionless local skin-friction coefficient

$D$ binary diffusion coefficient

Du Dufour number

$g$ acceleration due to gravity

$\mathrm{Gr}_{C}$ mass Grashof number

$G r_{T} \quad$ thermal Grashof number

$k_{T} \quad$ thermal diffusion ratio

$\overline{N u} \quad$ dimensionless average Nusselt number

$N u_{X} \quad$ dimensionless local Nusselt number

$\mathrm{Pr} \quad$ Prandtl number

$r$ radial coordinate

$r_{0} \quad$ radius of cylinder

$R$ dimensionless radial coordinate $t^{\prime} \quad$ time

$t$ dimensionless time

$T^{\prime} \quad$ temperature

$T_{m}{ }^{\prime}$ mean fluid temperature

$T$ dimensionless temperature

$u, v$ velocity components in $x, r$ directions respectively

$U, V$ dimensionless velocity components in $X, R$ directions respectively

$x \quad$ axial coordinate

$X$ dimensionless axial coordinate

$\alpha \quad$ thermal diffusivity

$\beta_{C} \quad$ volumetric coefficient of expansion with concentration

$\beta_{T} \quad$ volumetric coefficient of thermal expansion

$\rho$ density

$\eta \quad$ material constant

$\mu \quad$ viscosity of the fluid

$v \quad$ kinematic viscosity

Subscripts 


$\begin{array}{ll}S c & \text { Schmidt number } \\ \text { So } & \text { Soret number } \\ \overline{S h} & \text { dimensionless average Sherwood number } \\ S h_{X} & \text { dimensionless local Sherwood number }\end{array}$

\section{INTRODUCTION}

The double diffusive convection plays a significant role in various fields such as high quality crystal production, oceanography, production of pure medication, solidification of molten alloys, geothermally-heated lakes and magmas. For example the quality of the single crystal produced from the melts is limited by chemical and structural inhomogeneities. The defect generation depends on heat and mass transfer rates during solidification. When heat and mass transfer occur simultaneously in a moving fluid, the relations between the fluxes and the driving potentials are of more integrate nature. An energy flux can be generated not only by temperature gradients but also by concentration gradients. The energy flux caused by a concentration gradient is termed as the diffusion-thermo (Dufour) effect. On the other hand, mass fluxes can also be created by temperature gradients and this embodies the thermal-diffusion (Soret) effect. These fluxes are mainly governed by convective phenomena of the liquid phase during processing. In most of the studies that are related to heat and mass transfer process, Soret and Dufour effects are neglected on the basis that they are of smaller order of magnitude than the effects described by Fourier's and Fick's laws. But these effects are considered as second order phenomena and may become significant in areas such as hydrology, petrology, geosciences, etc. The Soret effect, for instance, has been utilized for isotope separation and in mixture between gases with very light molecular weight $\left(\mathrm{H}_{2}, \mathrm{He}\right)$ and of medium molecular weight $\left(\mathrm{N}_{2}\right.$, air). The Dufour effect was found to be of order of considerable magnitude such that it cannot be neglected (Eckeret and Drake, 1972). Dursunkaya and Worek (1992) studied diffusion-thermo and thermal-diffusion effects in transient and steady natural convection from a vertical surface, whereas Kafoussias and Williams (1995) presented the same effects on mixed convective and mass transfer steady laminar boundary layer flow over a vertical flat plate with temperature dependent viscosity. Postelnicu (2004) studied numerically the influence of a magnetic field on heat and mass transfer by natural convection from vertical surfaces in porous media considering Soret and Dufour effects. Seddeek (2004) has investigated the problem of thermaldiffusion and diffusion-thermo effects on the mixed free-forced convection and mass transfer in the presence of suction and blowing. Krishna Murthy et al. (2011) investigated the double diffusive free convection from a corrugated vertical surface in a darcy porous medium under Soret and Dufour effects. Recently, Reddy and Rao (2012) studied the heat and mass transfer characteristics of mixed convection about a circular cylindrical annulus in a porous medium, by taking into account the thermo-diffusion and diffusionthermo effects. w conditions on the wall

$\infty$ free stream conditions
With the growing importance of non-Newtonian fluids in modern technology and industries, the investigations on such fluids are desirable. Stokes (1966) generalized the classical Newtonian model to include the effect of couple-stresses in a way different from that of Eringen (1966). This is one among the several non-Newtonian fluid theories that are developed in the twentieth century. In his theory Stokes considered a body enclosing a volume without considering the microstructures of the infinitesimal fluid volume element. The set of all forces acting on an infinitesimal volume element is, in general, assumed to be equivalent to a single resultant force together with a resultant couple. The moment of the couple is assumed to be of non zero value. With this assumption Stokes has proposed the theory of couple-stress fluids allowing for the sustenance of couple-stresses in addition to the usual stresses. Also, in his theory, curvature twist rate tensor is proposed based on the pure kinematic aspects of rotation vector and couple-stress is defined in terms of this curvature twist rate tensor. Accordingly, in the balance of linear momentum of the couple-stress flow model, fourth order derivatives of velocities are involved and, hence, separate angular momentum equation need not be considered. These fluids can also sustain the existence of body forces as usual and in addition by body couples as well. The stress tensor is no longer symmetric in this theory. This couple-stress model has been widely used because of its great mathematical simplicity compared to that of the other models developed for the polar fluids. Recently, the study of couple-stress fluid flows has been the subject of great interest, due to its widespread industrial and scientific applications as in the case of micropolar fluids. Important field where couple-stress fluids have applications includes squeezing and lubrication (Chu et al., 2006; Lin, 1998; Naduvinamani and Patil, 2009; Chang-Jian et al., 2010), bio-fluidmechanics (Srivastava, 2003; Srivastava, 1986), MHD flows and synthesis and plasticity of chemical compounds. Another interesting application was studied by Umavathi and Malashetty (1999) for the flow and heat transfer characteristics of Oberbeck convection of a couple-stress fluid in a vertical porous stratum. Rudraiah and Chandrashekara (2010) investigated the effects of couple-stress fluid on the control of RayleighTaylor instability at the interface between a dense fluid accelerated by a lighter fluid. Recently, Rani et al. (2011) obtained the numerical solution for the transient free convective couple-stress fluid flow past a vertical cylinder.

From the above studies, it can be noted that the unsteady natural convective flow of a viscous incompressible couple-stress fluid over a vertical cylinder with Soret and Dufour effects has received very scant attention in the literature. Hence, in the present investigation our attention is focused on the 
boundary layer regime caused by a uniformly heated vertical cylinder, with constant wall temperature along the length of the cylinder, immersed in a couple-stress fluid with double diffusive effects. In section 2, a detailed description about the formulation of the problem is given. Also, the governing equations, such as mass, momentum, energy and concentration equations are derived and non-dimensionalized. In section 3, the details about the grid generation and numerical methods for solving the above governing equations are given. In section 4, transient twodimensional velocity, temperature and concentration profiles, average skin-friction, heat and mass transfer rates are analyzed. Also the comparison between the couple-stress fluid and Newtonian fluid flows are analyzed. Finally, the concluding remarks are made in section 5 .

\section{MATHEMATICAL FORMULATION}

An unsteady two-dimensional laminar combined heat and mass transfer boundary layer flow of a couplestress viscous incompressible fluid flow past an isothermal semi-infinite vertical cylinder of radius $r_{0}$ is considered as shown in Fig. 1. In addition, the Soret and Dufour effects are taken into account. The $x$-axis is measured vertically upward along the axis of the cylinder. The origin of $x$ is taken to be at the leading edge of the cylinder, where the boundary layer thickness is zero. The radial coordinate, $r$, is measured perpendicular to the axis of the cylinder. The surrounding stationary fluid temperature and concentration are assumed to be of ambient temperature $\left(T_{\infty}^{\prime}\right)$ and concentration $\left(C_{\infty}^{\prime}\right)$, respectively. Initially, i.e., at time $t^{\prime}=\mathrm{O}$ it is assumed that the cylinder and the fluid are of the same temperature $T_{\infty}^{\prime}$ and concentration $C_{\infty}^{\prime}$. As time increases $\left(t^{\prime}>0\right)$, the temperature and concentration of the cylinder is raised to $T_{w}^{\prime}\left(>T_{\infty}^{\prime}\right.$ ) and $C_{w}^{\prime}$ ( $>C_{\infty}^{\prime}$ ), respectively, and maintained at the same level for all time $t^{\prime}>0$. It is assumed that the effect of viscous dissipation is negligible in the energy equation since the flow velocity magnitude is expected to be small. The concentration $C^{\prime}$ of the diffusing species is assumed very small in the binary mixture and there is no chemical reaction between the diffusing species and the fluid. Under these assumptions, the boundary layer equations of mass, momentum, energy and concentration with Boussinesq's approximation are as follows:

$$
\begin{aligned}
& \frac{\partial(r u)}{\partial x}+\frac{\partial(r v)}{\partial r}=0 \\
& \rho\left(\frac{\partial u}{\partial t^{\prime}}+u \frac{\partial u}{\partial x}+v \frac{\partial u}{\partial r}\right)=\rho g \beta_{T}\left(T^{\prime}-T_{\infty}^{\prime}\right)+ \\
& \rho g \beta_{C}\left(C^{\prime}-C_{\infty}^{\prime}\right)+\frac{1}{r} \frac{\partial}{\partial r}\left(\mu r \frac{\partial u}{\partial r}\right)-\eta \nabla^{4} u \\
& \frac{\partial T^{\prime}}{\partial t^{\prime}}+u \frac{\partial T^{\prime}}{\partial x}+v \frac{\partial T^{\prime}}{\partial r}=\frac{\alpha}{r} \frac{\partial}{\partial r}\left(r \frac{\partial T^{\prime}}{\partial r}\right)+ \\
& \frac{D k_{T}}{c_{s} c_{p}} \frac{1}{r} \frac{\partial}{\partial r}\left(r \frac{\partial C^{\prime}}{\partial r}\right)
\end{aligned}
$$

$$
\begin{aligned}
& \frac{\partial C^{\prime}}{\partial t^{\prime}}+u \frac{\partial C^{\prime}}{\partial x}+v \frac{\partial C^{\prime}}{\partial r}=\frac{D}{r} \frac{\partial}{\partial r}\left(r \frac{\partial C^{\prime}}{\partial r}\right)+ \\
& \frac{D k_{T}}{T_{m}{ }^{\prime}} \frac{1}{r} \frac{\partial}{\partial r}\left(r \frac{\partial T^{\prime}}{\partial r}\right)
\end{aligned}
$$



Fig. 1. Schematic of the investigated problem along with the boundary conditions, where the notations are defined in Nomenclature.

where $\eta$ is a material constant with the dimension of momentum and describes the couple-stress fluid property. Usually, the ratio of material constants $\eta$ and $\mu$ has the dimensions of length square i.e $r_{0}{ }^{2}$ (see Stokes, 1984).

Stokes (1984) proposed mainly two types of boundary conditions, namely, the vorticity of the fluid on the boundary is equal to the rotational velocity of the boundary and the couple-stresses vanish on the boundary. The present problem is solved based on the earlier boundary condition. In view of this, the relevant initial and boundary conditions are given by:

$$
\begin{array}{cl}
t^{\prime} \leq 0: & u=0, v=0, T^{\prime}=T_{\infty}^{\prime}, C^{\prime}=C_{\infty}^{\prime} \quad \text { for all } x \text { and } r \\
t^{\prime}>0: & u=0, v=0, T^{\prime}=T_{w}^{\prime}, C^{\prime}=C_{w}^{\prime} \quad \text { at } \quad r=r_{0} \\
& u=0, \quad v=0, T^{\prime}=T_{\infty}^{\prime}, C^{\prime}=C_{\infty}^{\prime} \quad \text { at } \quad x=0 \\
& u \rightarrow 0, v \rightarrow 0, T^{\prime} \rightarrow T_{\infty}^{\prime}, C^{\prime} \rightarrow C_{\infty}^{\prime} \text { as } \quad r \rightarrow \infty
\end{array}
$$

and,

$\frac{\partial u}{\partial r}=\frac{\partial v}{\partial x} \quad$ at $\quad r=r_{0} \quad$ and as $r \rightarrow \infty$

By introducing the following non-dimensional quantities

$$
\begin{aligned}
& X=G r_{T}{ }^{-1} \frac{x}{r_{0}}, R=\frac{r}{r_{0}}, U=G r_{T}{ }^{-1} \frac{u r_{0}}{v}, \\
& V=\frac{v r_{0}}{v}, t=\frac{v t^{\prime}}{r_{0}{ }^{2}}, T=\frac{T^{\prime}-T_{\infty}^{\prime}}{T_{w}^{\prime}-T_{\infty}^{\prime}}, \\
& C=\frac{C^{\prime}-C_{\infty}^{\prime}}{C_{w}^{\prime}-C_{\infty}^{\prime}}, G r_{T}=\frac{g \beta_{T} r_{0}^{3}\left(T_{w}^{\prime}-T_{\infty}^{\prime}\right)}{v^{2}}, \\
& G r_{C}=\frac{g \beta_{C} r_{0}^{3}\left(C_{w}^{\prime}-C_{\infty}^{\prime}\right)}{v^{2}}, \\
& P r=\frac{\nu}{\alpha}, S c=\frac{\nu}{D}, B u=\frac{G r_{C}}{G r_{T}}, r_{0}=\left(\frac{\eta}{\mu}\right)^{\frac{1}{2}}
\end{aligned}
$$




$$
\begin{aligned}
& S o=\frac{D k_{T}\left(T_{w}{ }^{\prime}-T_{\infty}{ }^{\prime}\right)}{v T_{m}{ }^{\prime}\left(C_{w}{ }^{\prime}-C_{\infty}{ }^{\prime}\right)}, \\
& D u=\frac{D k_{T}\left(C_{w}{ }^{\prime}-C_{\infty}{ }^{\prime}\right)}{v c_{s} c_{p}\left(T_{w}{ }^{\prime}-T_{\infty}{ }^{\prime}\right)}
\end{aligned}
$$

(the symbols are explained in the nomenclature) in the Eqs. (1)-(4), the following equations are obtained:

$$
\begin{aligned}
& \frac{\partial U}{\partial X}+\frac{\partial V}{\partial R}+\frac{V}{R}=0 \\
& \frac{\partial U}{\partial t}+U \frac{\partial U}{\partial X}+V \frac{\partial U}{\partial R}=T+B u C+\frac{\partial U}{\partial R}\left(\frac{1}{R}-\frac{1}{R^{3}}\right)+ \\
& \frac{\partial^{2} U}{\partial R^{2}}\left(1+\frac{1}{R^{2}}\right)-\frac{2}{R} \frac{\partial^{3} U}{\partial R^{3}}-\frac{\partial^{4} U}{\partial R^{4}} \\
& \frac{\partial T}{\partial t}+U \frac{\partial T}{\partial X}+V \frac{\partial T}{\partial R}=\frac{1}{\operatorname{Pr}}\left(\frac{\partial^{2} T}{\partial R^{2}}+\frac{1}{R} \frac{\partial T}{\partial R}\right)+ \\
& \frac{\partial C}{\partial t}+U \frac{\partial C}{\partial X}+V \frac{\partial C}{\partial R}=\frac{1}{S c}\left(\frac{\partial^{2} C}{\partial R^{2}}+\frac{1}{R} \frac{\partial C}{\partial R}\right) \\
& S o\left(\frac{\partial^{2} T}{\partial R^{2}}+\frac{1}{R} \frac{\partial T}{\partial R}\right)
\end{aligned}
$$

The corresponding initial and boundary conditions in non-dimensional quantities are given by

$$
\begin{aligned}
& t \leq 0: U=0, V=0, T=0, C=0 \text { for all } X \text { and } R \\
& t>0: U=0, V=0, T=1, C=1 \quad \text { at } \quad R=1 \\
& U=0, V=0, T=0, C=0 \quad \text { at } \quad X=0 \\
& U \rightarrow 0, V \rightarrow 0, T \rightarrow 0, C \rightarrow 0 \quad \text { as } \quad R \rightarrow \infty
\end{aligned}
$$

Similarly, Eq. (6) in non-dimensional quantities is given by

$$
\frac{\partial U}{\partial R}=\frac{1}{G r_{T}{ }^{2}} \frac{\partial V}{\partial X} \text { at } R=1 \text { and as } R \rightarrow \infty
$$

\section{NUMERICAL SOLUTION}

In order to solve the unsteady coupled non-linear governing Eqs. (8)-(11) an implicit finite difference scheme of Crank-Nicolson type has been employed. To solve these equations, the region of integration is considered as a rectangle composed of the lines indicating $X_{\min }=0, X_{\max }=1, R_{\min }=1$ and $R_{\max }=20$, where $R_{\max }$ corresponds to $R=\infty$ which lies very far from the momentum, energy and concentration boundary layers. In order to obtain an economical and reliable grid system for the computations, a grid independency test has been performed and shown in Fig. 2. The steady-state velocity and temperature values obtained with the grid system of $100 \times 500$ differ in the second decimal place from those with the grid system of $50 \times 250$, and differ in the fifth decimal place from those with the grid system of $200 \times 1000$. Hence, the grid system of $100 \times 500$ has been selected for all subsequent analyses, with the mesh sizes in $X$ and $R$ directions taken as 0.01 and 0.03 , respectively. Also, the time step size dependency has been tested from which $\Delta t=0.01$ yielded a reliable result. The steadystate solution is assumed to have been reached when the absolute difference between the values of velocity, temperature as well as concentration at two consecutive time steps is less than $10^{-5}$ at all grid points. Also, this finite difference scheme is unconditionally stable and therefore, stability and compatibility ensure the convergence.



Fig. 2. Grid independency test for velocity, temperature and concentration profiles.

\section{RESUlt AND DisCUSSION}

For the validation, the temperature and concentration profiles of Newtonian fluids obtained by the current numerical procedure are compared with the existing results of Chen and Yuh (1980) for $S c=0.2, P r=0.7$, So $=0.0, D u=0.0$ and $B u=1.0$, as there are no experimental or analytical studies available to compare with the present problem. The current results are found to be in good agreement with the previous results available in literature as shown in Fig. 3.

In the present study, the Soret and Dufour effects are studied in detail for different values of $S o$ and $D u$, respectively, with fixed values of $\operatorname{Pr}$ [=0.71 (air)], $S c$ [ $=0.22]$ and $B u[=1.0]$ on the transient velocity, temperature and concentration profiles for couple-stress fluids. The value of the $S c$ is chosen to represent hydrogen in the air at approximately $25^{\circ} \mathrm{C}$ at $1 \mathrm{~atm}$ (Gebhart, 1971). The values of $S o$ and $D u$ are to be chosen in such a way that their product is constant according to their definition, provided that the $T_{m}{ }^{\prime}$ is constant i.e., (0.06) (Kafoussias and Williams, 1995). Also, in addition the results have been plotted for So = $\mathrm{Du}=0.0$. The simulated transient behaviour of the dimensionless velocity, temperature, concentration, average skin-friction coefficient and heat and mass transfer rates are discussed in detail in the succeeding subsections. 


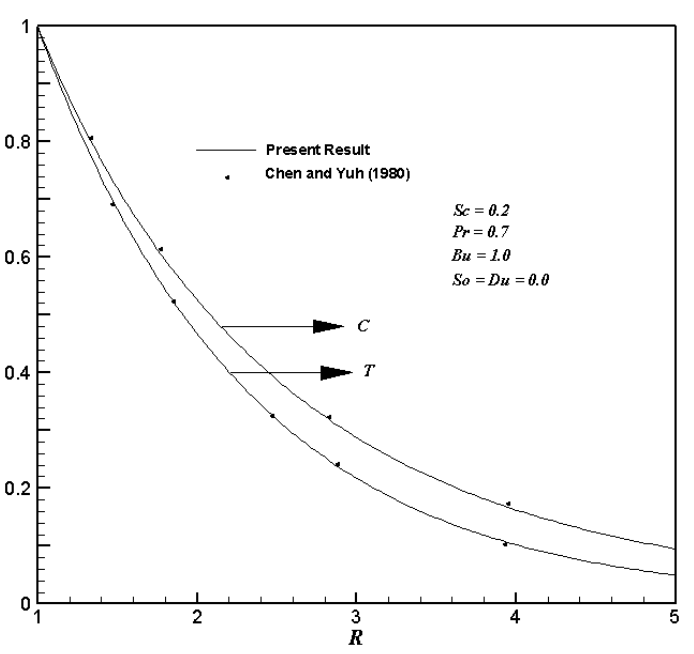

Fig. 3. Comparison of the temperature and concentration profiles for Newtonian fluids.

\subsection{Velocity $(U)$}

The simulated transient velocities $(U)$ at $(1,3.39)$ for different $S o$ and $D u$ against $t$ are shown graphically in Fig. 4(a). It is observed that the velocities increase with time monotonically from zero and reach temporal maxima, then decrease and at last reach the asymptotic steady-state. At the very early time (i.e., $t<<1$ ), the heat transfer is dominated by conduction. Shortly later, there exists a period when the heat transfer rate is influenced by the effect of convection with increasing upward velocities with time. When this transient period is almost ending and just before the steady-state is about to be reached, there exist overshoots of the velocities. From Fig. 4(b) it can be observed that velocity profiles reach their maximum value approximately at $(1,3.39)$. Similarly, the velocities at other locations also exhibit somewhat similar transient behavior. The time needed to reach the temporal maximum of the velocity increases with decreasing So or increasing $D u$. Also in the absence of Soret and Dufour effects $(\mathrm{So}=\mathrm{Du}=0)$, it is observed that the time required to reach the temporal maximum increases incomparison with the presence of Soret and Dufour effects.

Figure 4(b) shows the simulated steady-state velocity profiles against the $R$ at $X=1.0$. From this figure it is observed that the velocity profiles start with the value zero at the wall, reach their maxima and then monotonically decrease to zero along the radial coordinate. From Fig. 4(b) it is observed that in the vicinity of the wall the magnitude of the axial velocity is rapidly increasing as $R$ increases from $R_{\min }(=1)$. It is observed that for the decreasing $S o$ or increasing $D u$, the time to reach the steady-state increases slightly. Here, it can be noted that the velocity increases with increasing values of So. But the opposite trend is observed for the increasing $\mathrm{Du}$. Also, when $S o$ is high enough, the thermal and the solutal buoyancy forces combine their actions to enhance the convection velocity, which leads to the increase in the velocity of the fluid. If $\mathrm{So}=\mathrm{Du}=0$, the maximum velocity of a couple-stress fluid is decreased in comparison to that of the presence of Soret and Dufour effects. Also, if So = $\mathrm{Du}=0$, it is noticed that the time required to reach the steady-state increases in comparison to that of the presence of Soret and Dufour effects.
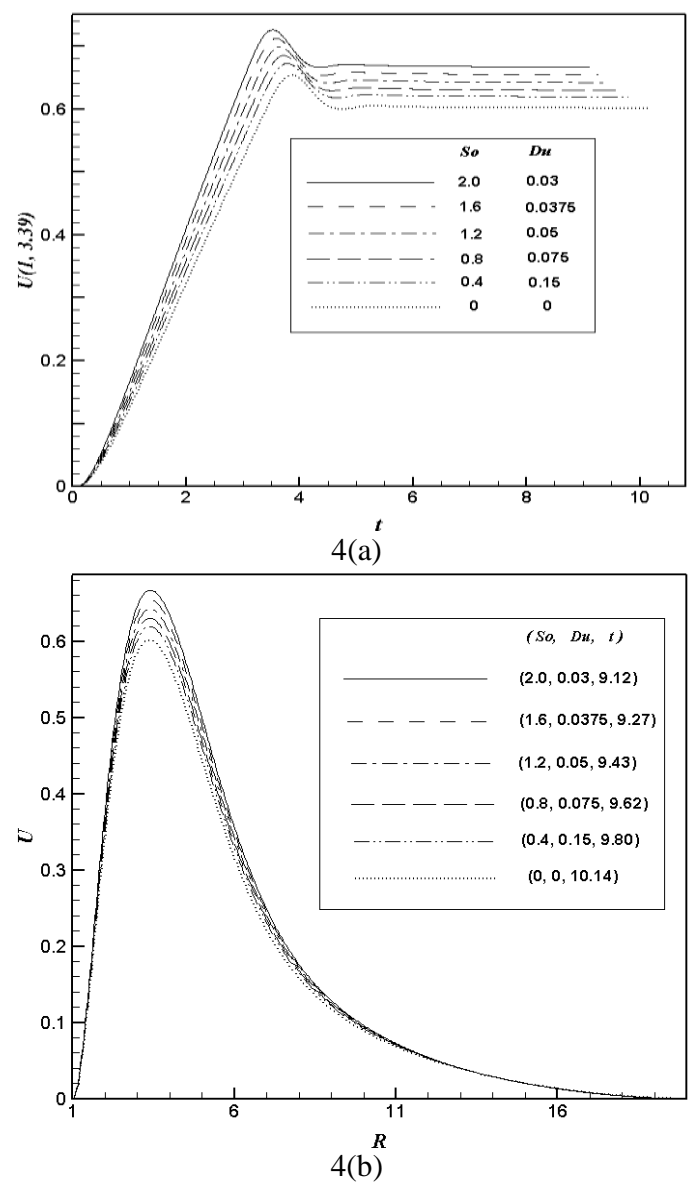

Fig. 4. The simulated (a) transient velocity at $(1,3.39)$; (b) steady-state velocity profile at $X=1.0$.

\subsection{Temperature (T)}

The simulated transient temperature $(T)$ for different $S o$ and $D u$ values with respect to $t$ are shown at the point $(1,1.22)$ in Fig. 5(a). Here, it is observed that these profiles increase with time, reach temporal maxima, decrease and again, after a slight increase, attain the steady-state asymptotically. The temperature at other locations also exhibit somewhat similar transient behavior. During the initial period, the nature of the transient temperature profiles is particularly noticeable. For all values of $S o$ and $D u$, the transient temperature profiles initially coincide and then deviate from each other after some time. Here, it is observed that the steady temperature value decreases with increasing So or decreasing $D u$. Also, if $\mathrm{So}=\mathrm{Du}=0$, the temporal maximum of the temperature increases in comparison to that of the existence of Soret and Dufour effects. This tendency is also found in Fig. 5(b) showing the simulated steady-state temperature profiles along the radial direction at $X=1.0$. Here, the temperature profiles start with the hot wall temperature $(T=1)$ and then monotonically decrease to zero along the radial coordinate. The time needed for the temperature to reach the steady-state increases as $D u$ increases or $S o$ decreases. Also, the time required to reach the temporal maximum of the temperature increases with the increasing $D u$ or decreasing So. It is noticed that the 
temperature increases with decreasing So, while the reverse trend is observed for the decreasing $D u$. This behavior is a direct consequence of the Soret effect, which produces a mass flux from lower to higher solute concentration driven by the temperature gradient.
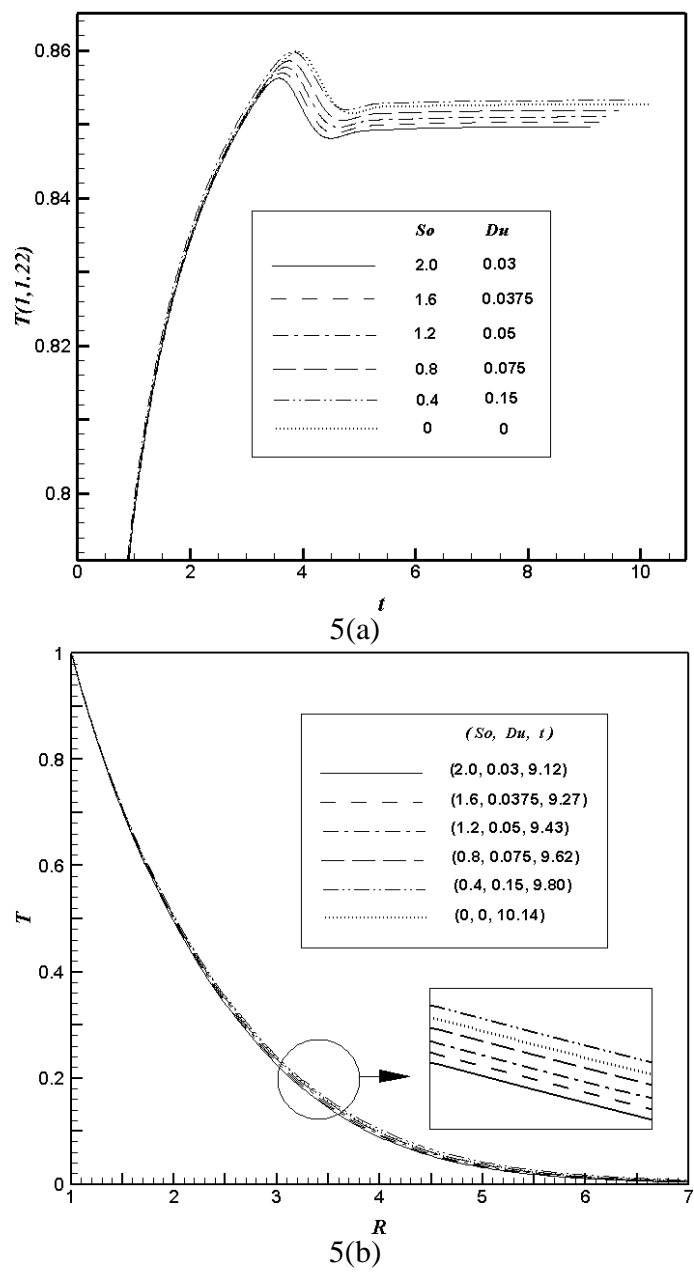

Fig. 5. The simulated (a) transient temperature at (1, 1.22 ); (b) steady-state temperature profile at $X=1.0$.

\subsection{Concentration $(C)$}

The simulated transient concentration $(C)$ for different values of $S o$ and $D u$ at the point $(1,1.34)$ against $t$ are shown in Fig. 6(a). Here, it is observed that these profiles increase at first with time, reach a temporal maximum, decrease and again, after slightly increasing, attain the steady-state asymptotically. The concentration at other locations also exhibit somewhat similar transient behavior. Here, it is seen that the steady concentration value increases with increasing So or decreasing $D u$. In the absence of Soret and Dufour effects $(\mathrm{So}=\mathrm{Du}=0)$, it is observed that the time required to reach the temporal maximum increases incomparison to that of the presence of Soret and Dufour effects. The simulated steady-state concentration profiles at $X=1.0$ along the $R$ direction are shown in Fig. 6(b). The concentration profiles start with the wall concentration $(C=1)$ and then monotonically decrease to zero along $R$. As $S o$ increases, the mass transfer rate decreases and hence the concentration profiles increase. But the opposite trend is observed as $D u$ increases. This is due to the fact that a larger So corresponds to a thicker concentration boundary layer relative to the momentum boundary layer. This results in a smaller concentration gradient near the cylinder. Also, it is observed that the time required for the concentration to reach the steady-state increases as $D u$ increases or $S o$ decreases. If $S o=D u=$ 0 , the maximum concentration of a couple-stress fluid is decreased in comparison to that of the presence of Soret and Dufour effects.
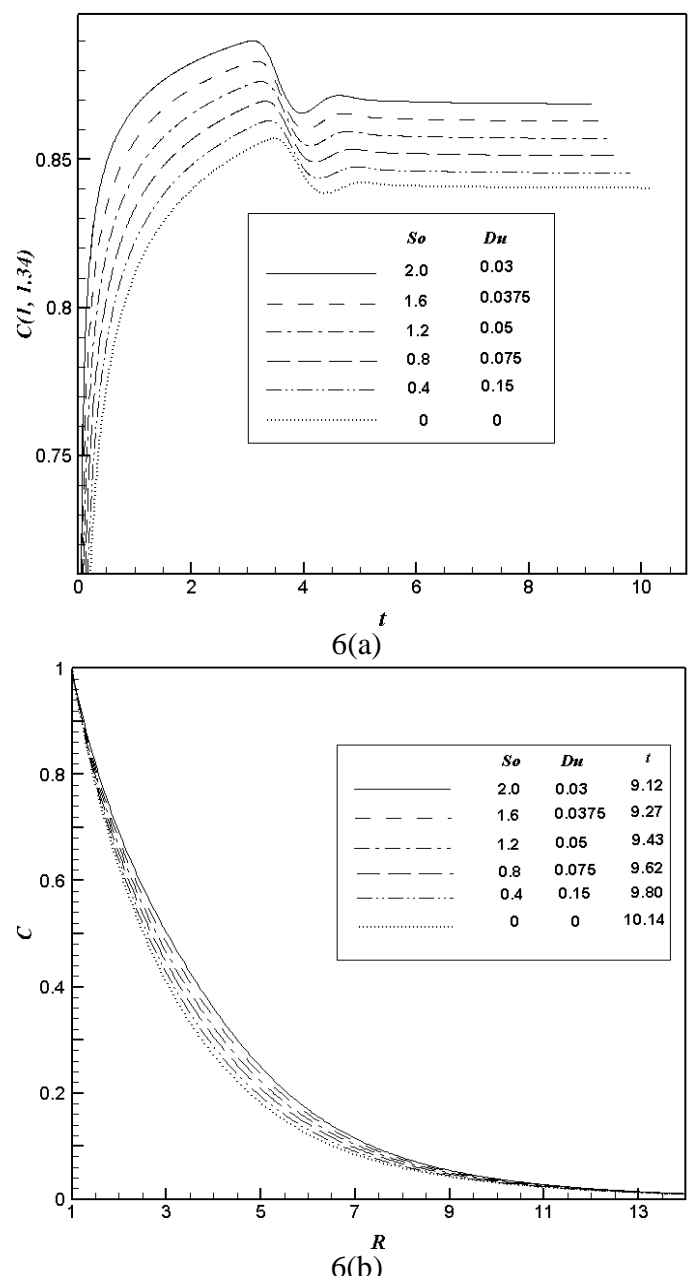

Fig. 6. The simulated (a) transient concentration at (1, 1.34); (b) steady-state concentration profile at $X=1.0$.

\subsection{Average Skin-friction Coefficient, Heat and Mass Transfer Rates}

Knowing the unsteady behavior of velocity, temperature and concentration profiles, it is worth to study the average skin-friction coefficient, average heat transfer rate (Nusselt number) and the average mass transfer rate (Sherwood number). The friction coefficient is an important parameter in the heat and mass transfer studies since it is directly related to the heat and mass transfer coefficients. Increased skin friction is generally a disadvantage in technical applications, while the increased heat and mass transfer can be exploited in some applications such as heat and mass exchangers, but should be avoided in others such as gas turbine applications, for instance. For the present problem these skin-friction coefficient, heat and mass 
transfer rates are derived and given in the following equations:

The wall shear stress at the wall can be expressed as

$$
\tau_{w}=\left(\mu \frac{\partial u}{\partial r}\right)_{r=r_{0}}
$$

By introducing the non-dimensional quantities given in Eq. (7), the Eq. (14) can be written as

$$
\tau_{w}=\frac{\mu^{2} G r_{T}}{\rho r_{0}^{2}}\left(\frac{\partial U}{\partial R}\right)_{R=1}
$$

Considering $\frac{\mu^{2} G r_{T}}{\rho r_{0}^{2}}$ to be the characteristic shear stress, the local skin-friction coefficient can be written as

$$
C_{f}=\left(\frac{\partial U}{\partial R}\right)_{R=1}
$$

The integration of the Eq. (16) from $X=0$ to $X=1$ gives the following average skin-friction coefficient.

$$
\overline{C_{f}}=\int_{0}^{1}\left(\frac{\partial U}{\partial R}\right)_{R=1} d X
$$

The local Nusselt number is given by

$$
N u_{x}=\frac{\dot{q}_{w} r_{0}}{k_{T}\left(T_{w}^{\prime}-T_{\infty}^{\prime}\right)},
$$

where the heat transfer, $\dot{q}_{w}$ is given by

$$
\dot{q}_{w}=-k_{T}\left(\frac{\partial T^{\prime}}{\partial r}\right)_{r=r_{0}}
$$

Thus, with the non-dimensional quantities introduced in Eq. (7), Eq. (18) can be written as

$$
N u_{X}=-\left(\frac{\partial T}{\partial R}\right)_{R=1}
$$

The integration of the above Eq. (19) with respect to $X$ from 0 to 1 yields the following average Nusselt number.

$$
\overline{N u}=-\int_{0}^{1}\left(\frac{\partial T}{\partial R}\right)_{R=1} d X
$$

The Sherwood number can be written as follows:

$$
S h_{x}=\frac{\dot{m}_{w} r_{0}}{D\left(C_{w}^{\prime}-C_{\infty}^{\prime}\right)},
$$

where the mass transfer, $\dot{m}_{w}$ is given by

$$
\dot{m}_{w}=-D\left(\frac{\partial C^{\prime}}{\partial r}\right)_{r=r_{0}}
$$

Thus, in the same way Eq. (21) can be written as

$$
S h_{X}=-\left(\frac{\partial C}{\partial R}\right)_{R=1}
$$

The integration of the above Eq. (22) with respect to $X$ yields the following average Sherwood number.

$$
\overline{S h}=-\int_{0}^{1}\left(\frac{\partial C}{\partial R}\right)_{R=1} d X
$$

The derivatives involved in Eqs. (17), (20) and (23) are evaluated by using a five-point approximation formula and then the integrals are evaluated by using the Newton-Cotes closed integration formula. The simulated average non-dimensional skin-friction coefficient, heat and mass transfer rates for couplestress fluids have been plotted against the time in Figs. 7-9 for different parameters, respectively.

The effects of $S o$ and $D u$ on the simulated average skinfriction coefficient are shown in Fig. 7. It is observed that for all values of $S o$ and $D u$ the average skin-friction coefficients increase at first with time, attain the peak values and, after slight decrease, reach asymptotically steady-state. Because the buoyancy-induced flow velocity is relatively low at the initial transient period, as seen in Fig. 4(a), the wall shear stress remain small, as shown in Fig. 7. However, the wall shear stress increases as the time proceeds, yielding an increase in the skin-friction coefficient. It is also observed that either by increasing $S o$ or by decreasing $D u$, the average skin-friction coefficient increases. If So $=\mathrm{Du}=0$, the average skin-friction is decreased in comparison to that of the presence of Soret and Dufour effects. This result lies in the same line with the velocity profiles plotted in Fig 4.

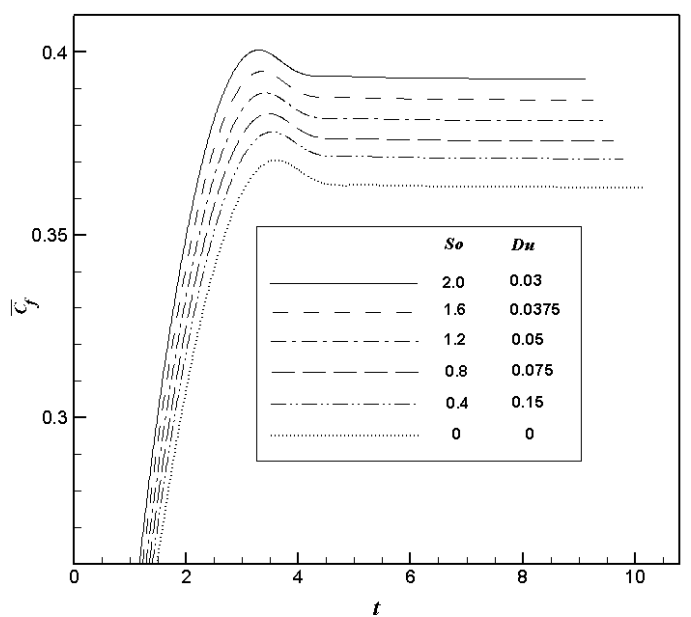

Fig. 7. The simulated average skin-friction.

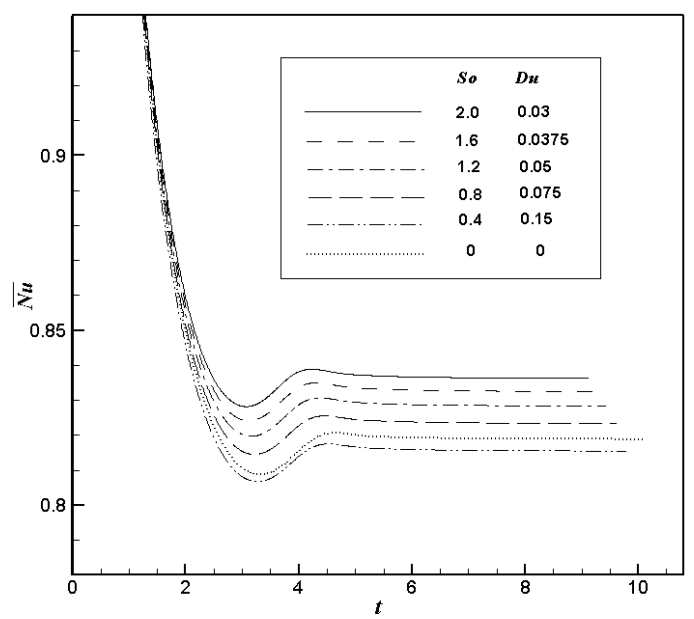

Fig. 8. The simulated average Nusselt number. 




Fig. 9. The simulated average Sherwood number.

In Fig. 8 the effects of $S o$ and $D u$ on the simulated average heat transfer rate are shown. It is observed that for short period of time after $t=0$, the average Nusselt numbers are almost the same for all values of $S o$ and $D u$. This shows that initially heat conduction is dominating compared with heat convection. It is seen that an increase in the value of $S o$ leads to a increase in the values of the average heat transfer rate. Increasing So speeds up the spatial decay of the temperature near the heated surface together with increased flow velocity near the wall, yielding an increase in the rate of heat transfer. While the reverse trend is observed for increasing values of $D u$. If So $=\mathrm{Du}=0$, the trend of average heat transfer rate is similar to that of the presence of Soret and Dufour effects. In Fig. 9, the effects of $S o$ and $D u$ on the simulated average mass transfer rate are presented. Figure 9 shows that during an initial period of time mass diffusion is dominating compared to mass convection. For all values of $S o$ and $D u$, the average Sherwood number decreases monotonically, then after slight fluctuation, reaches the asymptotic steady-state. It is noticed that the average mass transfer rate increases with decreasing $S o$ or increasing $D u$. If $S o=D u=0$, the average Sherwood number is increased in comparison to that of the presence of Soret and Dufour effects.

\subsection{Comparison between the couple-stress and Newtonian fluids}

Figure 10 illustrates the steady-state velocity, temperature and concentration contours for couplestress and Newtonian fluid flows with fixed $S o=2.0$ and $D u=0.03$. From Figs. 10(a) and Figs. 10(b) it is observed that the maximum velocity occurs at $X=1.0$. This is the reason that the simulated steady-state velocity, temperature and concentration profiles are plotted at $X=1.0$ in Figs. (4)-(6). It can be noticed that from Figs. 10(a) and 10(b), the velocity of a couplestress fluid is smaller compared to that of the Newtonian fluid. This is due to the fact that in couplestress fluid flow there are additive diffusion terms (biharmonic term) compared with the Newtonian fluid (refer Eq. (9)). Also, from Figs. 10(a) and 10(b) it is observed that the steady-state velocity, temperature and concentration contours of couple-stress fluid are different from that of the Newtonian fluid. Thus, it can be concluded that the steady-state velocity, temperature and concentration profiles for the couple-stress fluid flow differ from those of the Newtonian fluid flow.

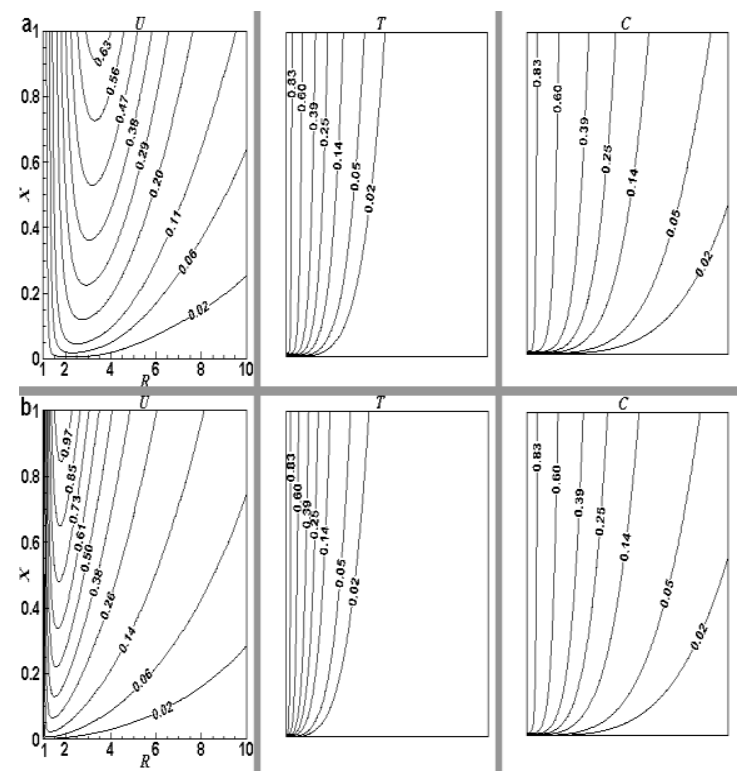

Fig. 10. Steady-state velocity, temperature and concentration contours with $S o=2.0$ and $D u=0.03$ for

(a) couple-stress fluid; (b) Newtonian fluid.

\section{CONCLUSION}

A numerical study has been carried out for the transient natural convection boundary layer flow of a couplestress viscous incompressible fluid over a semi-infinite vertical cylinder with thermal-diffusion and diffusionthermo effects. The governing equations are derived and normalized based on the length dependent effect introduced by the couple-stress fluid flow where the biharmonic operator is involved. A Crank-Nicolson type of implicit method is used to solve the system of coupled governing equations together with the tridiagonal and pentadiagonal algorithms. The computations are carried out for the values of So $(=$ 2.0, 1.6, 1.2, 0.8, 0.4 and 0.0$)$ and $D u(=0.03,0.0375$, $0.05,0.075,0.15$ and 0.0 ).

From the present study, it is observed that for the velocity, temperature and concentration profiles, the time elapsed to reach the temporal maximum and the steady-state increases with the increasing $D u$ or decreasing So. Also, if $S o=D u=0$, it is noticed that the time required to reach the steady-state increases in comparison to that of the presence of Soret and Dufour effects. It is noticed that as $S o$ increases or $D u$ decreases, velocity and concentration profiles are increasing, but the temperature decreases. The influence of $S o$ or the $D u$ is less on the temperature profiles in comparison with the velocity and concentration profiles. The concentration and Sherwood number are more affected by So. Also, the average values of the skin-friction and Nusselt number increases as $S o$ increases or $D u$ decreases. While the opposite trend is observed for the Sherwood number. Particularly, this study reveals that the results pertaining to the couplestress fluid differ significantly from those of the Newtonian fluid. The deviations of velocity, temperature and concentration profiles of couple-stress fluid flow from those of the Newtonian fluid flow turn out to be considerable. 
Though the present study deals only with laminar flows, this work can be extended to studies on turbulent flows. Also, the body forces like electromagnetic force arising from the MHD flows and the body couples arising in the momentum equation due to the couple-stress fluid can be taken into consideration. Also, the present model can be expanded into studies of flow past plates, wedges, cones, and spheres etc., based on required applications.

\section{ACKNOWLEDGEMENTS}

The authors are thankful to the reviewers for their valuable suggestions and comments.

\section{REFERENCES}

Carnahan, B., H.A. Luther and J.O. Wilkes (1969). Applied numerical methods. John Wiley Sons, New York.

Chang-Jian, C., J. Chen and H. Yau (2010). Nonlinear dynamic analysis of a hybrid squeeze-film dampermounted rigid rotor lubricated with couple-stress fluid and active control. Appl. Math. Modell. 34, 2493-2507.

Chen, T.S. and C.F. Yuh (1980). Combined heat and mass transfer in natural convection along a vertical cylinder. Int. J. Heat and Mass Transfer 23, 451461.

Chu, H.M., S.Y. Hu and W.L. Li (2006). Effects of couple-stresses on pure squeeze EHL motion of circular contacts. J.Mech. 22(1), 77-84.

Dursunkaya, Z. and W.M. Worek (1992). Diffusionthermo and thermal diffusion effects in transient and steady natural convection from a vertical surface. Int. J. Heat and Mass Transfer 35, 20602065 .

Eckeret, E.R.G. and R.M. Drake (1972). Analysis of heat and mass transfer. McGraw Hill, New York.

Eringen, A.C. (1966). Theory of micropolar fluids. $J$. Math. and Mech. 16, 1-18.

Gebhart, B. (1971). Heat transfer. McGraw-Hill, New York.

Kafoussias, N.G. and N.G. Williams (1995). Thermaldiffusion and diffusion-thermo effects on mixed free-forced convective and mass transfer boundary layer flow with temperature dependent viscosity. Int. J. Engg. Sci,. 33, 1369-1384.

Krishna Murthy, S.V.S.S.N.V.G., P. Chandra, M. Nigam, B.V. Rathish-Kumar and V. Sangwan, (2011). A study of double diffusive free convection from a corrugated vertical surface in a darcy porous medium under Soret and Dufour effects. ASME J. Heat Transfer, 133, 601-607.

Lin, J. (1998). Squeeze film characteristics of finite journal bearings: couple-stress fluid model. Tribology International, 31(4), 201- 207.

Naduvinamani, N.B. and S.B. Patil (2009). Numerical solution of finite modified Reynolds equation for couple-stress squeeze film lubrication of porous journal bearings. Computers and Structures, 87, 1287-1295.

Postelnicu, A. (2004). Influence of a magnetic field on heat and mass transfer by natural convection from vertical sufaces in porous media considering Soret and Dufour effects. Int. J. Heat and Mass Transfer, $47,1467-1475$.

Rani, H.P., G. Janardhana Reddy and C.N. Kim (2011). Numerical analysis of couple-stress fluid past an infinite vertical cylinder. Engineering applications of computational fluid mechanics, 5(2), 159-169.

Reddy, P.S. and V.P. Rao (2012). Thermo-Diffusion and Diffusion - Thermo Effects on convective heat and mass transfer through a porous medium in a circular cylindrical annulus with quadratic density temperature variation - Finite element study. J. appl. Fluid Mech., 5(4), 139-144.

Rudraiah, N. and G. Chandrashekara (2010). Effects of couple-stress on the growth rate of RayleighTaylor instability at the interface in a finite thickness couple-stress fluid. J. appl. Fluid Mech,. 3(1), 83-89.

Seddeek, M.A. (2004). Thermal-diffusion and diffusion-thermo effects on mixed free-forced convective flow and mass transfer over an accelerating surface with a heat source in the presence of suction and blowing in the case of variable viscosity, Acta Mechanica,172, 83-94.

Srivastava, L.M. (1986). Peristaltic transport of a couple-stress fluid, Rheol Acta, 25, 638-641.

Srivastava, V.P. (2003). Flow of a couple-stress fluid representing blood through stenotic vessels with a peripheral layer. Indian J. Pure and Appl. Math. 34(12), 1727-1740.

Stokes, V.K. (1984). Theories of Fluids with Microstructure. Springer-Verlag, New York Tokyo. 
H. P. Rani and G. J. Reddy / JAFM, Vol. 6, No. 4, pp. 545-554, 2013.

Stokes, V.K. (1966). Couple-stress in fluids. Physics of Fluids 9, 1709-1715.

Umavathi, J.C. and M.S. Malashetty (1999). Oberbeck convection flow of a couple-stress fluid through a vertical porous stratum. Int. J. Non-Linear Mech. $34,1037-1045$.
Von Rosenberg, D.U. (1969). Methods for the Numerical Solution of Partial Differential Equations. American Elsevier Publishing Company, New York. 